http://dx.doi.org/10.18359/ravi.2088

\title{
Competencias directivas, un reto para la educación superior ${ }^{1}$
}

\author{
Alba Ruth Vargas Montealegre ${ }^{2} \&$ Paula Andrea García Ortiz ${ }^{3}$ \\ Universidad de Ibagué, Colombia
}

Recibido, julio 18 de 2016

Concepto evaluación, diciembre 19 de 2016

Aceptado, diciembre 27 de 2016
Referencia: Vargas Montealegre, A.; García Ortiz, P.

(2017). "Competencias directivas, un reto para la educación superior". Revista Academia y Virtualidad, 10, (1), 11-22

\section{Resumen}

La presente investigación busca determinar el nivel de importancia de las competencias genéricas y específicas del administrador financiero, tomando como referente el Proyecto Tuning en Latinoamérica-Europa y el Proyecto GRIICA Colombia. Su enfoque es mixto, apoyado en técnicas estadísticas multivariantes; para ello, se utilizaron cuestionarios y entrevistas aplicadas a estudiantes, graduados y docentes del programa de Administración Financiera de la Universidad de Ibagué. Los hallazgos permiten evidenciar competencias específicas en componentes integrales, tales como administración y organizaciones, mercadeo, finanzas, emprendimiento, economía y evaluación y formulación de proyectos, como también las principales competencias genéricas agrupadas en autoevaluación y toma de decisiones, aprendizaje autónomo y comunicación intercultural. Al respecto, se encontraron similitudes y diferencias significativas entre los grupos involucrados para, finalmente, sugerir un conjunto de competencias por considerar en los planes de estudio de este programa e invitar a generar nuevos casos de estudio con el fin de validar o fortalecer el modelo utilizado.

Palabras clave: competencias, administración financiera, Proyecto GRIICA, Proyecto Tuning, plan de estudio.

\footnotetext{
${ }^{1}$ Artículo de investigación científica y tecnológica.

${ }^{2}$ Docente tiempo completo, Universidad de Ibagué. alba.vargas@unibague.edu.co.

${ }^{3}$ Integrante Grupo de Investigación UNIDERE, Universidad de Ibagué. paulagarcia1293@gmail.com.
} 


\title{
Managerial skills-A challenge for higher education
}

\begin{abstract}
This research aims to outline the relevance of generic and specific skills by financial manager related to the Tuning project in Latin America-Europe and the Colombian GRIICA Project. A mixed focus was supported by multivariate statistical techniques. For this purpose, questionnaires and interviews were applied to students, graduates and teachers of the Financial Management program at the Universidad de Ibagué. Findings allow us to highlight specific skills for integral constituents, such as management and organizations, marketing, finance, entrepreneurship, economics, and projects evaluation and design as well as the main generic skills grouped in self-evaluation and decision-making, autonomous learning, and crosscultural communication. In this regard, we found significant similarities and differences among groups involved to finally suggest a set of skills to consider within curricula of this program, and request new case studies generated in order to validate or strengthen the model used.
\end{abstract}

Keywords: skills, financial management, GRIICA Project, Tuning Project, curriculum.

\section{Competências diretivas, um repto para a educação superior}

\section{Resumo}

Na presente pesquisa procura-se determinar o nível de importância das competências genéricas e especificas do administrador financeiro, tendo como referente ao projeto Tuning em Latino América-Europa e ao Projeto GRIICA Colômbia. Eu enfoque é misturado, apoiado nas técnicas estadísticas multivariântes; para isso, utilizaram-se questionários e entrevistas aplicadas a estudantes, graduados e docentes do programa de Administração Financeira da Universidade de Ibagué. Os achados permitem evidenciar competências especificas nos componentes integrantes tais como administração e organizações, mercado, finanças, empreendimento, economia e avaliação e formulação de projetos, como também as principais competências genéricas agrupadas em auto avaliação e tomada de decisões, aprendizagem autónomo e comunicação intercultural. Ao respeito encontraram-se similitudes e diferencias significativas entre os grupos involucrados para, al final, sugerir um conjunto de competências por considerar nos planos de estudos deste programa e invitar a gerar novos casos de estudo a fim de validar ou fortalecer o modelo utilizado.

Palavras chave: competências, administração financeira, Projeto GRIICA, Projeto Tuning, plano de estudo. 


\section{Introducción}

Las tendencias gerenciales enmarcadas en el contexto global en el que están inmersas las organizaciones, como los cambios tecnológicos, sociales, políticos y económicos, requieren habilidades propias del administrador, tanto para liderar procesos como para emprender nuevos negocios, lo que ha exigido que a nivel mundial se generen espacios para reflexionar sobre la educación superior y su función en la formación de los futuros directivos para el desarrollo de competencias requeridas para conformar negocios o incursionar en el mercado laboral.

En consecuencia, en Europa se implementó el Proyecto Tuning, un gran reto para las instituciones, por cuanto generó la creación de un entorno de trabajo para que los académicos lograran llegar a puntos de referencia, de comprensión y de confluencia sobre las competencias que se deberían desarrollar los administradores financieros (González \& Wagenaar, 2003), aspectos tenidos en cuenta por GRIICA Colombia, caso ASCOLFA, 2008-2010 (Castrillón Cifuentes \& Cabeza de Vergara, 2010), que consolidó los estudios de América Latina y Europa, de tal manera que permitieran identificar las competencias del administrador, siendo un referente para la identificación de las competencias.

La metodología utilizada para el presente estudio se basó en el método GRIICA Colombia - Tuning, cuya investigación tiene un enfoque mixto, donde se utilizaron técnicas multivariantes como elanálisis de componentes principales y la prueba no paramétrica de Kruskal-Wallis. Se emplearon instrumentos como la entrevista y el cuestionario para conocer la percepción de las competencias. La población estuvo constituida por estudiantes, docentes y graduados. Por último, la muestra probabilística se conformó aleatoriamente, según registros de bases de datos del programa, tomados de los años 2012-2015.

Los resultados permitieron determinar las competencias genéricas y específicas con base en los componentes de la administración, y desde el análisis de los hallazgos se muestran las similitudes y diferencias más significativas entre la percepción de los docentes en contraste con las percepciones de estudiantes y graduados, resaltando la necesidad de detectar los requerimientos reales del campo laboral para incorporar las competencias en los planes de estudio de los programas de administración financiera, para que los futuros graduados puedan responder a las demandas del entorno y así alcanzar una mayor movilidad académica y profesional.

\section{Metodología}

Con base en el proyecto general en el que participan las universidades que integran el capítulo Centro ASCOLFA, este estudio, de enfoque mixto, se aborda a partir de técnicas multivariantes en su método cuantitativo, y en cuanto al método cualitativo se fundamenta en la descripción de resultados.

Asimismo, la población estuvo conformada por estudiantes, egresados o graduados $\mathrm{y}$ docentes del programa de Administración Financiera, encontrados en los registros de las bases de datos de la Universidad de Ibagué, con corte al semestre A de 2015. La muestra representativa se constituyó mediante técnicas probabilísticas con un margen de error del 5\%, con base en los siguientes criterios

- Estudiantes del programa de Administración Financiera, matriculados de tercero a octavo semestre de formación.

- Graduados, registrados en los últimos tres años.

- Docentes que orientan asignaturas según componentes de formación en administración y finanzas.

En correspondencia, se especifica la muestra utilizada, así: 
Tabla 1. Muestra utilizada para el estudio.

\begin{tabular}{cc}
\hline Actores & N \\
\hline Graduados & 38 \\
Estudiantes & 52 \\
Docentes & 14 \\
\hline Total & $\mathbf{1 0 4}$ \\
\hline \multicolumn{2}{c}{ Fuente: Elaboración propia. }
\end{tabular}

Como fuentes de información secundaria, se contó con los informes de GRIICA Colombia, Proyecto Tuning Europa y América Latina, Ministerio de Educación de Colombia, con información histórica del programa, entrevistas con los directores del programa de los últimos 10 años y artículos consultados en bases de datos.

Adicionalmente, el instrumento de investigación utilizado para este estudio, fue validado por el estudio de GRIICA Colombia y las competencias de los componentes fueron determinados según un panel de expertos de las universidades integrantes del capítulo Centro ASCOLFA, los cuales participan en la red que formuló el proyecto de investigación (Universidades de Amazonia, Tolima, Cooperativa e Ibagué), del cual forma parte este estudio, y fue adaptada por los autores para su aplicación a los diferentes actores del Programa de Administración Financiera, según prueba piloto.

Asimismo, para estudio se determinaron 10 variables de caracterización demográfica, 35 ítems relacionados con competencias genéricas y 36 ítems relacionados con competencias específicas, para lo cual se empleó una medición en escala de Likert de 1 a 4, en términos del grado de importancia, siendo (1) nada y (4) mucho.

Para reducir la dimensionalidad del conjunto de las 35 competencias genéricas medidas con datos ordinales en escala de Likert, se empleó un análisis de componentes principales categóricos con el método de normalización principal por variable, con lo cual se buscó determinar el número de dimensiones o variables latentes que pudieran representar el conjunto de competencias genéricas. En cuanto a las competencias específicas, se realizó una agrupación de acuerdo con el componente o área al cual pertenecen (administración, mercados, emprendimiento, economía, finanzas, formulación de proyectos) para posteriormente realizar la comparación de los resultados arrojados entre cada grupo de interés contemplado en el estudio.

Finalmente, se analizaron los coeficientes alfa de Cronbach para cada uno de los factores extraídos y se realizó una prueba de Kruskal-Wallis para definir si la percepción de las competencias genéricas y específicas (agrupadas en factores) de los grupos encuestados (estudiantes, egresados y docentes) son iguales o significativamente diferentes.

De esta manera se muestra en los resultados las competencias más representativas para cada grupo de involucrados (docentes, estudiantes y graduados) por grado de importancia, posteriormente las competencias de mayor coincidencia entre docentes, graduados y estudiantes y a partir de éste análisis se sugieren competencias genéricas y específicas a considerar en los programas de Administración Financiera.

\section{Resultados}

A continuación, se describen los principales resultados obtenidos en la investigación, para evaluar el nivel de importancia de 
competencias genéricas en tres grupos establecidos y competencias específicas en torno a seis áreas o componentes: administración y organizaciones; mercadeo; emprendimiento; economía; finanzas; formulación y evaluación de proyectos; desde la perspectiva de los estudiantes, egresados y docentes del programa de Administración Financiera.

\section{Competencias genéricas}

A partir de los resultados obtenidos en el análisis de componentes categóricos pueden extraerse 3 factores que explican el 55,9\% de la varianza común (tabla 2). Se han excluido del análisis la competencia genérica 33 (espíritu emprendedor) debido a que presentaba cargas altas en los factores 1 y 2 , al igual que la competencia genérica 24 por presentar cargas altas en los factores 1 y 3 . También fue excluida la competencia genérica 34 (cuidado del cuerpo) por presentar cargas muy bajas para todos los factores.

A continuación se condensan en la tabla 2 los resultados obtenidos en cada uno de los factores y las competencias que los conforman.

Tabla 2. Resultados análisis de componentes principales categóricos sobre la percepción de la importancia de las competencias genéricas

\begin{tabular}{|c|c|c|c|c|}
\hline \multirow[b]{2}{*}{ Ítem } & \multirow[b]{2}{*}{ Competencias genéricas } & \multicolumn{3}{|c|}{ Factor } \\
\hline & & $\begin{array}{c}1 \\
\text { Competencias de } \\
\text { autoevaluación y toma de } \\
\text { decisiones }\end{array}$ & $\underset{\substack{2 \\
\text { Competeneias de } \\
\text { aprendizaje autónomo }}}{2}$ & $\begin{array}{c}3 \\
\text { Competencias de } \\
\text { comunicación } \\
\text { intercultural }\end{array}$ \\
\hline $\mathrm{CG} 22$ & $\begin{array}{l}\text { Valoración y respeto por la diversidad y } \\
\text { multiculturalidad }\end{array}$ &, 777 &, 052 &,- 126 \\
\hline CG18 & Habilidades interpersonales &, 751 &, 056 &,- 080 \\
\hline CG13 & $\begin{array}{l}\text { Capacidad para actuar en nuevas } \\
\text { situaciones }\end{array}$ &, 726 &,- 049 &,- 124 \\
\hline CG19 & $\begin{array}{l}\text { Capacidad de motivar y conducir hacia } \\
\text { metas comunes }\end{array}$ & ,698 &,- 040 &,- 090 \\
\hline CG4 & $\begin{array}{l}\text { Conocimientos sobre el área de estudio y } \\
\text { la profesión }\end{array}$ & ,667 &,- 028 &,- 049 \\
\hline CG12 & Capacidad crítica y autocrítica & ,664 &, 097 &,- 056 \\
\hline CG15 & $\begin{array}{l}\text { Capacidad para identificar, plantear y } \\
\text { resolver problemas }\end{array}$ & ,652 &,- 233 &, 334 \\
\hline CG30 & Pensamiento matemático & ,652 &,- 230 &,- 190 \\
\hline CG21 & Compromiso con su medio socio-cultural & ,649 &,- 076 &,- 089 \\
\hline CG9 & Capacidad de investigación & ,631 &,- 125 &, 004 \\
\hline CG31 & Cultura cientifica y tecnológica & ,614 &,- 261 &,- 201 \\
\hline CG25 & $\begin{array}{l}\text { Capacidad para formular y gestionar } \\
\text { proyectos }\end{array}$ & ,610 & 237 &,- 166 \\
\hline CG28 & Comunicación en lengua materna &, 595 &,- 131 &,- 407 \\
\hline CG8 & $\begin{array}{l}\text { Habilidades en el uso de las tecnologias } \\
\text { de la información y la comunicación }\end{array}$ &, 588 &,- 136 &, 122 \\
\hline CG3 & $\begin{array}{l}\text { Capacidad para organizar y planificar el } \\
\text { tiempo }\end{array}$ &, 567 &,- 228 &,- 116 \\
\hline CG14 & Capacidad creativa & ,557 &,- 172 &,- 218 \\
\hline CG2 & $\begin{array}{l}\text { Capacidad de aplicar los conocimientos } \\
\text { en la práctica }\end{array}$ &, 547 &, 272 & ,116 \\
\hline CG5 & $\begin{array}{l}\text { Responsabilidad social y compromiso } \\
\text { ciudadano }\end{array}$ &, 540 &,- 260 &, 336 \\
\hline CG1 & $\begin{array}{l}\text { Capacidad de abstracción, análisis y } \\
\text { sintesis }\end{array}$ &, 535 &,- 235 &,- 103 \\
\hline CG26 & Compromiso ético &, 533 &, 144 &,- 274 \\
\hline CG16 & Capacidad para tomar decisiones &, 508 &, 292 &,- 135 \\
\hline CG35 & Pensamiento sistémico & ,485 &, 250 &,- 160 \\
\hline $\mathrm{CG} 27$ & Compromiso con la calidad & ,442 &,- 070 &,- 374 \\
\hline CG20 & $\begin{array}{l}\text { Compromiso con la preservación del } \\
\text { medio ambiente }\end{array}$ & ,425 &,- 133 &,- 275 \\
\hline CG32 & Ética y ciudadanía & ,296 &, 880 & 346 \\
\hline CG17 & $\begin{array}{l}\text { Capacidad de trabajo en equipo } \\
\text { intercultural }\end{array}$ & ,296 &, 879 & ,348 \\
\hline CG11 & $\begin{array}{l}\text { Habilidades para buscar, procesar y } \\
\text { analizar información procedente de } \\
\text { fuentes diversas }\end{array}$ &, 312 &, 875 &, 347 \\
\hline
\end{tabular}




\begin{tabular}{|c|c|c|c|c|}
\hline CG10 & $\begin{array}{l}\text { Capacidad de aprender y actualizarse } \\
\text { permanentemente }\end{array}$ &, 312 &, 871 &, 355 \\
\hline CG23 & $\begin{array}{l}\text { Habilidad para trabajar en contextos } \\
\text { internacionales }\end{array}$ & 290 &,- 423 & ,806 \\
\hline CG29 & $\begin{array}{l}\text { Comprensión lectora en inglés } \\
\text { comunicación en segunda lengua }\end{array}$ &, 281 &,- 430 & ,806 \\
\hline CG6 & Capacidad de comunicación oral y escrita &, 317 &,- 420 & ,803 \\
\hline CG7 & $\begin{array}{l}\text { Capacidad de comunicación en un } \\
\text { segundo idioma }\end{array}$ &, 244 &,- 279 & ,687 \\
\hline & Autovalor & 9,54 & 4,46 & 3,89 \\
\hline & Varianza explicada & $29,81 \%$ & $13,96 \%$ & $12,16 \%$ \\
\hline & Alfa de Cronbach &, 924 &, 801 &, 767 \\
\hline
\end{tabular}

El primer factor (autovalor 9,54) representa el $29,81 \%$ de la varianza común e incluye 24 competencias genéricas, las cuales presentan una excelente consistencia interna $(\alpha=, 924)$. Este factor se ha denominado "Competencias de autoevaluación y toma de decisiones" por estar compuesto por aquellas habilidades y destrezas que todo profesional requiere para tomar decisiones responsables consigo mismo y con los demás.

El segundo factor (autovalor 4,46) representa el $13,96 \%$ de la varianza común e incluye 4 competencias genéricas, las cuales presentan una buena consistencia interna $(\alpha=, 801)$, el cual es denominado "Competencias de aprendizaje autónomo" por estar conformado por aquellas habilidades que el profesional en administración desarrolla para su formación autónoma y continua.

Por último, el tercer factor (autovalor 3,89) representa el $12,16 \%$ de la varianza común e incluye 4 competencias genéricas, las cuales presentan una aceptable consistencia interna $(\alpha=, 767)$. Fue nombrado "Competencias de comunicación intercultural", puesto que comprende elementos fundamentales para la adecuada participación en situaciones comunicativas específicas, acontecidas bajo un contexto que puede ser internacional.

En consecuencia, se procedió a imputar por medio del paquete estadístico AMOS el valor de los factores, a partir de las competencias genéricas correspondientes a cada uno de ellos. Posteriormente, debido a la no normalidad de los factores fue necesario realizar la prueba no paramétrica de Kruskal-Wallis (tabla 3) para definir si la percepción de las competencias genéricas (agrupadas en factores) de los grupos encuestados (estudiantes, egresados $\mathrm{y}$ docentes) son iguales o significativamente diferentes.

Tabla 3. Prueba de Kruskal-Wallis para los factores de competencias genéricas

\begin{tabular}{cccc}
\hline & $\begin{array}{c}\text { Competencias de autoevaluación y toma } \\
\text { de decisiones }\end{array}$ & $\begin{array}{c}\text { Competencias de aprendizaje } \\
\text { autónomo }\end{array}$ & $\begin{array}{c}\text { Competeneias de comunicación } \\
\text { intercultural }\end{array}$ \\
\hline Chi-cuadrado & 8,490 & 11,868 & 4,478 \\
Gl & 2 & 2 & 2 \\
Sig. asintótica &, $014^{*}$ &, $003^{*}$ &, 107 \\
\hline
\end{tabular}

a. Variable de agrupación: Grupo encuestados*. p<0,05 Fuente: Elaboración propia. 
Los resultados de la prueba de KruskalWallis exhibidos en la tabla 3 indican que existe una diferencia estadísticamente significativa para los grupos de competencias genéricas de "Autoevaluación y toma de decisiones" y las competencias genéricas de "Aprendizaje autónomo" entre los distintos grupos encuestados (estudiantes, egresados, docentes). Es decir, la percepción de la importancia que tienen esas competencias genéricas para el perfil de los administradores financieros no es igual para los estudiantes, egresados y docentes. En contraste, las competencias genéricas de "Comunicación intercultural" no presentan diferencias significativas entre los grupos encuestados y, por tanto, la percepción de la importancia de estas competencias es similar.

Después de determinar la existencia de una diferencia significativa entre los grupos encuestados, fue necesario realizar un análisis post hoc para identificar si la diferencia proviene de los 3 grupos encuestados o solamente de la diferencia entre 2 grupos. De esta manera, se realizaron 3 pruebas de Kruskal-Wallis (tabla 4), al comparar diferentes parejas de grupos encuestados, encontrando que no existe una diferencia significativa entre la percepción de los estudiantes y egresados para ningún factor de competencias genéricas. No obstante, si se perciben diferencias significativas entre la percepción de los docentes y estudiantes para los factores de "Competencias de autoevaluación y toma de decisiones" y "Competencias de aprendizaje autónomo", así como diferencias entre la percepción de los docentes y egresados para el factor de "Competencias de aprendizaje autónomo", las cuales se relacionan con las habilidades que debe tener el administrador para desenvolverse en un contexto académico o empresarial.

Tabla 4. Prueba post hoc de Kruskal-Wallis para los factores de competencias genéricas

\begin{tabular}{|c|c|c|c|}
\hline Grupos & Co & mpetencias genéricas & \\
\hline $\begin{array}{l}\text { Estudiantes- } \\
\text { Egresados }\end{array}$ & $\begin{array}{c}\text { Competencias de autoevaluación y } \\
\text { toma de decisiones }\end{array}$ & $\begin{array}{l}\text { Competencias de } \\
\text { aprendizaje autónomo }\end{array}$ & $\begin{array}{c}\text { Competeneias de comunicación } \\
\text { intercultural }\end{array}$ \\
\hline Chi-cuadrado & 2,772 & 3,398 & 2,732 \\
\hline Sig. Asintótica &, 096 &, 065 &, 098 \\
\hline $\begin{array}{l}\text { Estudiantes- } \\
\text { Docentes }\end{array}$ & $\begin{array}{l}\text { Competencias de autoevaluación y toma } \\
\text { de decisiones }\end{array}$ & $\begin{array}{l}\text { Competeneias de aprendizaje } \\
\text { autónomo }\end{array}$ & $\begin{array}{l}\text { Competencias de comunicación } \\
\text { intercultural }\end{array}$ \\
\hline Chi-cuadrado & 8,153 & 10,302 & 3,195 \\
\hline Sig. Asintótica &, $004^{*}$ &, $001^{\text {شे }}$ &, 074 \\
\hline $\begin{array}{l}\text { Egresados- } \\
\text { Docentes }\end{array}$ & $\begin{array}{l}\text { Competencias de autoevaluación y toma } \\
\text { de decisiones }\end{array}$ & $\begin{array}{l}\text { Competencias de aprendizaje } \\
\text { autónomo }\end{array}$ & $\begin{array}{l}\text { Competencias de comunicación } \\
\text { intercultural }\end{array}$ \\
\hline Chi-cuadrado & 1,767 & 4,871 & ,056 \\
\hline Sig. Asintótica &, 184 &, $027^{\star}$ &, 813 \\
\hline
\end{tabular}

Los anteriores resultados demuestran que es importante considerar las aportaciones de estudiantes y egresados en la configuración de las competencias en los planes de estudio, para adecuar la oferta de trabajo y los requerimientos de la demanda, tal y como lo expresan Bartual \& Turmo (2016). De modo que se articule el conocimiento disciplinar de los docentes con las necesidades reales de los sectores económicos. En donde es clave reconocer las competencias laborales como herramientas que demuestran la capacidad real del saber hacer las cosas, y su influencia en el desempeño efectivo de un trabajo, expresado por Freire, Teijeiro \& Pais (2011). 


\section{Competencias específicas}

Para el análisis de las competencias específicas, éstas fueron agrupadas por áreas o componentes de la siguiente manera:

\section{Administración y organizaciones}

- Desarrollar un planeamiento estratégico, táctico y operativo.

- Identificar y optimizar los procesos de negocio de las organizaciones.

- Desarrollar, implementar y gestionar sistemas de control administrativo.

- Identificar las interrelaciones funcionales de la organización.

- Evaluar el marco jurídico aplicado a la gestión empresarial.

- Ejercer el liderazgo para el logro y consecución de metas en la organización.

- Administrar y desarrollar el talento humano en la organización.

- Identificar aspectos éticos y culturales de impacto recíproco entre la organización y el entorno social.

- Mejorar e innovar los procesos administrativos.

- Utilizar las tecnologías de información y comunicación en la gestión.

- Administrar la infraestructura tecnológica de una empresa.

- Formular y optimizar sistemas de información para la gestión.

\section{Mercados}

- Administrar un sistema logístico integral.

- Ejercer el liderazgo para el logro y consecución de metas en la organización.

- Detectar oportunidades para emprender nuevos negocios y/o desarrollar nuevos productos.

- Utilizar las tecnologías de información y comunicación en la gestión.

- Formulación de planes de marketing

\section{Emprendimiento}

- Identificar y optimizar los procesos de negocio de las organizaciones.
- Detectar oportunidades para emprender nuevos negocios y/o desarrollar nuevos productos.

- Elaborar, evaluar y administrar proyectos empresariales en diferentes tipos de organizaciones

- Detectar oportunidades para emprender nuevos negocios y/o desarrollar nuevos productos

\section{Economía}

- Identificar y administrar los riesgos de negocios de las organizaciones

- Identificar aspectos éticos y culturales de impacto recíproco entre la organización y el entorno social.

- Detectar oportunidades para emprender nuevos negocios $\mathrm{y} / \mathrm{o}$ desarrollar nuevos productos.

\section{Finanzas}

- Identificar y administrar los riesgos de negocios de las organizaciones

- Elaborar, evaluar y administrar proyectos empresariales en diferentes tipos de organizaciones

- Interpretar la información contable y la información financiera para la toma de decisiones gerenciales.

- Usar la información de costos para el planeamiento, el control y la toma de decisiones.

- Tomar decisiones de inversión, financiamiento y gestión de recursos financieros en la empresa.

\section{Proyectos}

- Identificar y administrar los riesgos de negocios de las organizaciones.

- Elaborar, evaluar y administrar proyectos empresariales en diferentes tipos de organizaciones.

- Interpretar la información contable y la información financiera para la toma de decisiones gerenciales.

- Usar la información de costos para el 
planeamiento, el control y la toma de decisiones.

Posteriormente, con estas competencias agrupadas en componentes, se procedió imputar el valor de cada variable latente por medio del paquete estadístico AMOS de acuerdo con las competencias específicas que conforman cada factor. Debido a la no normalidad de los factores fue necesario realizar la prueba no paramétrica de Kruskal-Wallis para definir si las percepciones de las competencias específicas (agrupadas en factores) de los grupos encuestados (estudiantes, egresados y docentes) fueron iguales o significativamente diferentes.

Tabla 5. Prueba de Kruskal-Wallis para los factores de competencias especificas

\begin{tabular}{|c|c|c|c|c|c|c|}
\hline & \multicolumn{6}{|c|}{ Competencias específicas } \\
\hline & Administración & Mercadeo & Finanzas & Emprendimiento & Economia & Ev, proyectos \\
\hline $\begin{array}{c}\text { Chi- } \\
\text { cuadrado }\end{array}$ & 8,402 & 6,036 & 5,553 & 1,206 & 5,630 & 4,712 \\
\hline Gl & 2 & 2 & 2 & 2 & 2 & 2 \\
\hline $\begin{array}{c}\text { Sig. } \\
\text { asintótica }\end{array}$ &, $015^{*}$ & ,049* &, 062 &, 547 &, 060 & 095 \\
\hline
\end{tabular}

Tal como se muestra en la tabla 5, existe una diferencia estadísticamente significativa para los grupos de competencias específicas de administración y mercadeo entre los distintos grupos encuestados (estudiantes, egresados, docentes). Es decir, la percepción de la importancia que tienen esas competencias específicas para el perfil de los administradores financieros no es igual para los estudiantes, egresados y docentes.

Al igual que en el análisis de las competencias genéricas, se realizó un análisis post hoc para identificar si la diferencia significativa entre los grupos involucrados se debe al efecto de todos los grupos o solamente a la diferencia entre dos de ellos, encontrando los siguientes resultados.

Tabla 6. Prueba post hoc de Kruskal-Wallis para los factores de competencias especificas

\begin{tabular}{|c|c|c|c|c|c|c|}
\hline Grupos & & & Competen & as específicas & & \\
\hline $\begin{array}{l}\text { Estudiantes- } \\
\text { Egresados }\end{array}$ & Administración & Mercadeo & Finanzas & Emprendimiento & Economia & $\begin{array}{c}\text { Ev. } \\
\text { proyectos }\end{array}$ \\
\hline Chi-cuadrado & 871 & 1,317 & 1,107 &, 089 &, 543 &, 273 \\
\hline Sig. asintótica &, 351 &, 251 & .293 & .766 &, 461 & .601 \\
\hline $\begin{array}{c}\text { Estudiantes- } \\
\text { Docente }\end{array}$ & Administración & Mercadeo & Finanzas & Emprendimiento & Economia & $\begin{array}{c}\text { Ev. } \\
\text { proyectos }\end{array}$ \\
\hline Chi-cuadrado & 5.439 & 3,237 & 2,769 &, 711 & 2,865 & 2,527 \\
\hline Sig. asintótica &, $020^{*}$ & 072 & ,096 &, 399 & ,091 &, 112 \\
\hline $\begin{array}{l}\text { Egresados- } \\
\text { Docentes }\end{array}$ & Administración & Mercadeo & Finanzas & Emprendimiento & Economía & $\begin{array}{c}\text { Ev. } \\
\text { proyectos }\end{array}$ \\
\hline Chi-cuadrado & 8,386 & 5,679 & 5,686 & 1,359 & 6,772 & 5,882 \\
\hline Sig. asintótica &, $004^{*}$ &, $017^{\star}$ &, 017 " & .244 &, $009^{\star}$ &, $015^{\star}$ \\
\hline
\end{tabular}


Así, la tabla 6 presenta 3 pruebas de KruskalWallis, al comparar distintas parejas de grupos encuestados, encontrándose que no existe una diferencia significativa entre la percepción de los estudiantes y egresados para ningún factor de competencias específicas. Sin embargo, si se perciben diferencias significativas entre estudiantes-docentes para las competencias específicas de administración, así como diferencias significativas entre egresados-docentes para todos los grupos de competencias específicas excepto para el factor de emprendimiento.

\section{Discusión}

Si bien estudios como CINDA y ASCOLFAGRIICA (2010) sirvieron como base para establecer los fundamentos por evaluar y proporcionar una identificación de las competencias genéricas y específicas, al igual que los hallazgos de la investigación de Castrillón, Cabeza \& Lombana (2015), con el Proyecto Tuning América Latina, que lograron aprobar, jerarquizar y clasificar las competencias de la disciplina administrativa en Colombia, desde la visión de empleadores $\mathrm{y}$ académicos, el presente estudio otorga la mirada de docentes, estudiantes y graduados y los hallazgos permiten evidenciar las competencias por componente académico, considerando así una perspectiva más integrada.

En efecto, los resultados de análisis de competencias genéricas, mostraron tres componentes principales. El primero de ellos se denomina competencias de autoevaluación y toma de decisiones, que involucra variables como capacidad de toma de decisiones, abstracción, análisis y síntesis, comunicación oral y escrita, la capacidad de trabajo en equipo, cultura científica y tecnológica, habilidades en el uso de las tecnologías de la información y la comunicación, entre otras. En concordancia con los preceptos de Capó, Expósito \& Masiá (2007) y Castro Valles (2015), quienes resaltan la importancia de la información en el entorno empresarial actual, porque modifica en gran manera las posibilidades de hacer negocio más allá de las tradicionales limitaciones de espacio y de tiempo. Además, las TIC están cambiando a gran velocidad la forma de trabajar, de comprar, de hacer negocios y de comunicarse (Nadler \& Tushman, 2000). Igualmente, García (2006) argumenta que los anteriores elementos son fundamentales para el desarrollo de un alumno universitario.

El segundo factor correspondiente al aprendizaje autónomo, considera aspectos relevantes como capacidad de aprender y actualizarse permanentemente; capacidad de trabajo en equipo intercultural; habilidades para buscar, procesar y analizar información procedente de fuentes diversas; capacidad de aprender y actualizarse permanentemente; lo cual reafirma los planteamientos exhibidos por De la Cruz (2003), quien destaca el carácter activo, autónomo, generador de nuevos conocimientos y reflexivo del alumno universitario, que busca aprender el conocimiento útil y práctico, para ser más competitivo en el mercado laboral y lograr su adecuada incorporación (Ruiz, 2006).

El tercer componente, denominado "Competencias de comunicación intercultural", comprende la relevancia del trabajo en contextos internacionales, comprensión lectora en inglés, capacidad de comunicación oral y escrita, entre otras. Lo que coincide con la visión de Rehaag (2010) y Porter \& Kramer (2011), quienes indican que el conocimiento de un segundo idioma contribuye a la comunicación e interacción con personas de otras culturas y facilita el desarrollo profesional.

Los docentes, egresados y estudiantes reconocieron las competencias específicas como aquellas disciplinares que indudablemente son invariables y que deben constituir el perfil profesional del egresado, incluyendo en sus consideraciones elementos como optimización de procesos, liderazgo, emprendimiento, TIC e información contable, como una competencia integral, una unidad que implica elementos del conocimiento que deben ser dominados en su totalidad y no solo alguna de sus partes, armonizando con los argumentos de Blanco, 
Morales \& Torre(2008) y Gonczi (1997).

Así mismo es importante continuar con el desarrollo curricular y un trabajo del equipo docente para el diseño de actividades de enseñanza y aprendizaje, que garanticen la adquisición de estas competencias en el aula, lo cual podría establecer las bases para futuras investigaciones.

Este trabajo presenta una limitación fundamental. La realización del estudio se efectuó en una universidad y en un programa de formación, por lo que quizá los resultados tengan una aplicabilidad limitada, y probablemente no sean generalizables.

\section{Conclusiones}

A través del tiempo, son variados los estudios que se han elaborado con respecto del tema de competencias genéricas y específicas, fragmentándolas en actitudes y habilidades. Sin embargo, no consideran aspectos fundamentales para cada programa educativo y los generalizan sin tener en cuenta las dinámicas y el campo de acción de cada profesión, especialmente en el área administrativa, lo que ha generado confusión al momento de clasificar las competencias y adecuarlas a las necesidades del entorno.

Conforme a los resultados, se determinaron las competencias generales más representativas desde la perspectiva de los involucrados, como son poseer un espíritu emprendedor, compromiso con la calidad, capacidad de comunicación oral y escrita, capacidad de tomar decisiones, capacidad de resolver problemas compromiso ético y capacidad de abstracción, análisis y síntesis.

En cuanto a las competencias específicas constituida por los componentes "Administración y organizaciones; Mercados; Emprendimiento; Economía; Finanzas; Formulación y evaluación de proyectos", no se encontraron diferencias significativas entre la percepción de los estudiantes y egresados. Sin embargo, sí se perciben diferencias significativas entre estudiantesdocentes para las competencias específicas de "Administración y organizaciones" así como diferencias significativas entre egresados-docentes para todos los grupos de competencias específicas excepto para el factor "Emprendimiento" constituido por "Identificar y optimizar los procesos de negocio de las organizaciones; detectar oportunidades y emprender nuevos negocios; elaborar, evaluar y administrar proyectos de negocios de las organizaciones".

Es necesario que en el momento de desarrollar un nuevo plan de estudios y continuar con la acreditación de alta calidad para los programas, se tenga muy en cuenta la capacidad de aplicar los conocimientos adquiridos en la práctica como principal competencia genérica, aspecto expresado por egresados, lo cual les puede garantizar mayor éxito en el mercado laboral.

Finalmente, la constante necesidad de innovación y búsqueda de nuevas habilidades que imperan en las dinámicas de la globalización, exigen que las competencias identificadas se integren en los componentes de los planes curriculares, como también, sean objeto de estudio en los programas de formación, para mantener su actualización. Es imperativo incluir, en futuros estudios, a los empresarios, quienes fueron descartados en el desarrollo del análisis, por la dificultad en la consecución de la información requerida y la obtención de un número de empresas inferior a la esperada en la muestra autoseleccionada

\section{Referencias}

Bartual, M., \& Turmo, J. (2016). Educación superior y competencias para el empleo. El punto de vista de los empresarios. Revista Complutense de Educación, 27(3). https://doi. org/10.5209/rev_RCED.2016.v27.n3.47645

Blanco, Á., Morales, P., \& Torre, J. C. (2008). Formación Universitaria basada en competencias. En Á. Blanco, P. Morales, J. C. Torre, \& L. Prieto Navarro (Ed.), La enseñanza universitaria centrada en el aprendizaje. Estrategias útiles para el profesorado (pág. 188). Madrid, España: Publicaciones UAM. 
Capó Vicedo, J., Expósito Langa, M., \& Masiá Buades, E. (2007). La importancia de los cluster para la competitividad de las PYME en una economía global. Revista EURE, 33(98), 119-133.

Castrillón Cifuentes, J., \& Cabeza de Vergara, L. (2010). Las competencias de los administradores en Colombia a la luz del Proyecto Tuning América Latina 20082010. Bogotá DC, Cundinamarca, Colombia: Publicaciones ASCOLFA.

Castrillón Cifuentes, J., Cabeza de Vergara, L., \& Lombana, J. (2015). Competencias más importantes para la disciplina administrativa en Colombia. Contaduría y Administración, 60(4), 776-795. doi: http://doi.org/10.1016/j. cya.2015.07.009

Castro Valles, A. (2015). Competencias administrativas y académicas en el profesorado de educación media superior. Revista Mexicana de Investigación Educativa, 20(64), 263-294.

De La Cruz, A. (2003). El proceso de convergencia europeo: Ocasión de modernizar la universidad española si se produce un cambio de mentalidad en gestores, profesores y estudiantes. Aula Abierta, 82, 191-216.

Freire, M., Teijeiro, M., \& Pais, C. (2011). La adecuación entre las competencias adquiridas por los graduados y las requeridas por los empresarios: The match between graduateacquired and employer-required competencies. Ministerio de Educación. Recuperado a partir de https://books.google.com/books?hl=en\&lr $=\& \mathrm{id}=\mathrm{KHP} 2 \mathrm{CQAAQBAJ} \&$ oi $=$ fnd $\& p g=\mathrm{PA} 2$ $6 \& d q=\% 22$ de + entrada: $+07-02-2011+$ Fecha + de+aceptaci\%C3\%B3n:\%22+\%22the+last + fe $\mathrm{w}+$ years + the + Spanish+university+system+ha $\mathrm{s}+$ been + forced $\% 22+\% 22 \mathrm{The}+$ study + covered $+1,052+$ graduates + sorted + by + competency $+a$ ffinity $\% 22+\&$ ots $=$ v6SSVfvQNI\&sig $=$ nMaRh zEkvwUwb9rkZ-AYfqW2SAE
García, M. R. (16 de Noviembre de 2006). Las competencias de los alumnos universitarios. Revista Interuniversitaria de Formación del Profesorado, 20(3), 253-269.

Gonczi, A. (1997). Problemas asociados con la implementación de la educación basada en la competencia: de lo atomístico a lo holístico. Formación basada en competencia laboral. CINTERFORT-OIT, 165-166.

González, J., \& Wagenaar, R. (. (2003). Tuning educational structures in Europe. Informe final fase I. Universidad de Deusto - Universidad de Groningen. Bilbao (España): Publicaciones Universidad de Deusto. Obtenido de http:// www.ub.edu/cubac/sites/default/files/tuning educational_structures_espanyol_0.pdf

Medina, A., \& Domínguez, M. C. (2003). Los procesos de observación del prácticum: análisis de competencias. Revista española de pedagogía (233), 75-76.

Mora, J. G. (2003). Competencias y empleo de los jóvenes graduados universitarios. Revista de Educación, 330, 157-170.

Nadler, D., \& Tushman, M. (2000). La empresa del futuro: imperativos estratégicos y competencias básicas para el siglo XXI. Harvard Deusto Business Review (96), 34-46.

Porter, M., \& Kramer, M. (2011). Creating Shared Value. How to reinvent capitalism and unleash a wave of innovation and growth. Harvard Business Review, 1-17.

Rehaag, I. (Marzo-Abril de 2010). La perspectiva intercultural en la educación. El Cotidiano (160), 75-83. Obtenido de http://www.redalyc.org/articulo. oa? id=32512766009

Ruiz, R. G. (2006). Las competencias de los alumnos universitarios. Revista interuniversitaria de formación del profesorado, (57), 253-270. 